\title{
FIRST RESULTS OF MICROWAVE MEASUREMENT OF BROADBAND RADIO INTERFERENCE FILTERS
}

\author{
Karel Hoffmann and Zbynek Skvor \\ Czech Technical University in Prague, Dpt. Electromagnetic Field \\ Technicka 2, 16627 Praha 6, Czech Republic, voice:+420-224 352276 \\ e-mail: hoffmann@,fel.cvut.cz, skvor@fel.cvut.cz
}

\begin{abstract}
A vector measurement method for testing of multiport interference filters for shielded chambers has been used. Measured data up to $3 \mathrm{GHz}$ are presented. Authors believe that this is the first time such results are published.
\end{abstract}

\section{Introduction:}

Modern communication devices as well as clock frequencies of desktop computers are climbing well into gigahertz region. Preventing electromagnetic interference and/or information leakage gets more and more important and difficult at the same time.

Some premises handle data so important that there is a strong need for data protection against leakage caused by radiofrequency leakage. Such premises are often shielded and all lines connecting these premises with the rest of world should be safeguarded against leakage. This applies not only to data transmission lines, but also to power network lines. These lines are blocked using radiofrequency filters. Such devices are often designed to handle up to hundred kilowatts@50 Hz, and therefore provide poor environment for microwave measurement.

Although measurements are required up to $3 \mathrm{GHz}$, there is no methodology available for this band. The main difference between radiofrequency interference filters and "classic" microwave devices is that the filters do not possess microwave connectors.

Therefore a proper connection of the filter with coaxial vector network analyzer ports must be arranged and a well defined reference plane chosen.

The purpose of this paper is to present an approach to this problematic and the first experimental results with three types of such interference filters.

\section{Experimental arrangement}

Figs. 1. to 3. show three types of interference filters with mounting boxes opened. With respect to mechanical arrangement of the filters it was decided to place the measurement reference planes close to the lid of the mounting boxes and to develop special adapters between $50 \Omega$ coaxial line and the reference planes. 
According to [1], filter measurement should be carried out repeatedly, with terminating impedances 0.1, 50 and $100 \mathrm{Ohms}$. There are no VNAs on the market providing for such impedance requirements. In order to facilitate such measurements, broadband adapters should be used. An adapter to $0.1 \mathrm{Ohm}$ is not attainable, so $50: 1 \mathrm{Ohm}$ one was chosen instead. In order to maintain broad band, resistive transitions have been designed, see Figs. 4 . to 8.

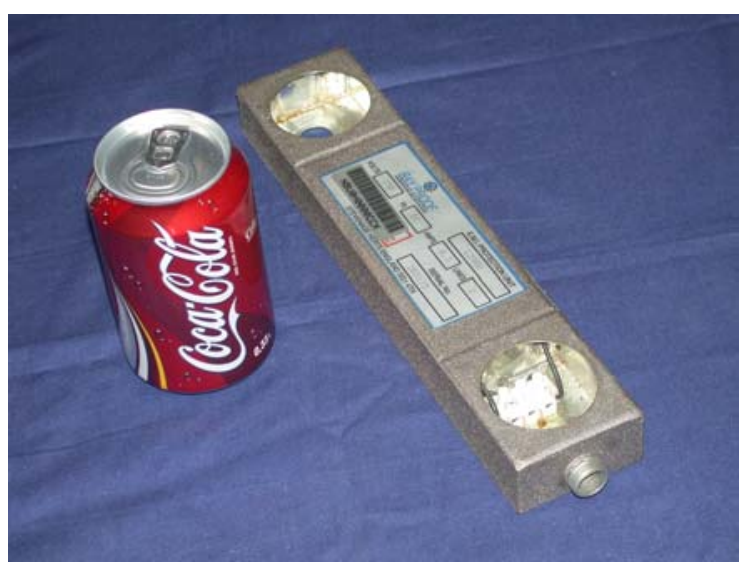

Fig. 1. Small, data line filter

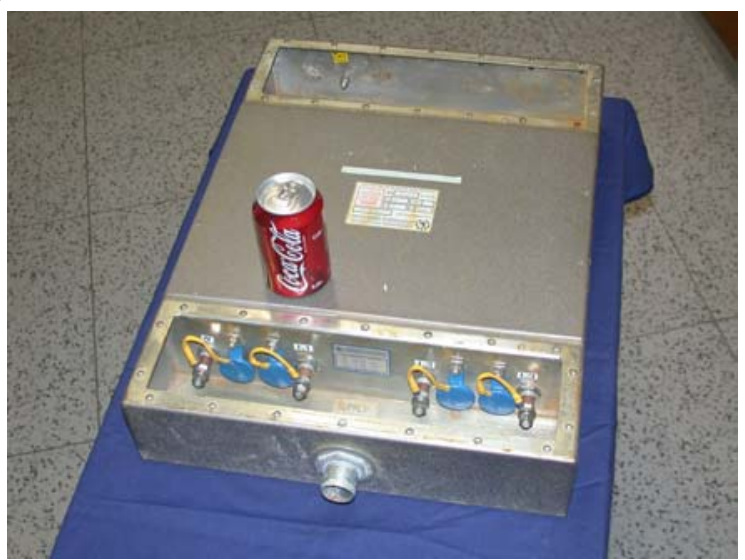

Fig. 3. Big, high- power 3 phase filter

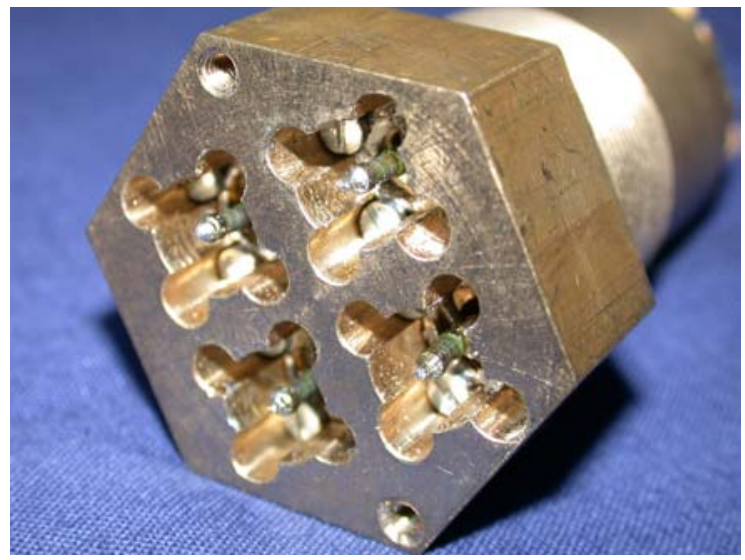

Fig. 5. 50 $\Omega-100 \Omega$, adapter - resistive elements uncovered, for three-phase filters.

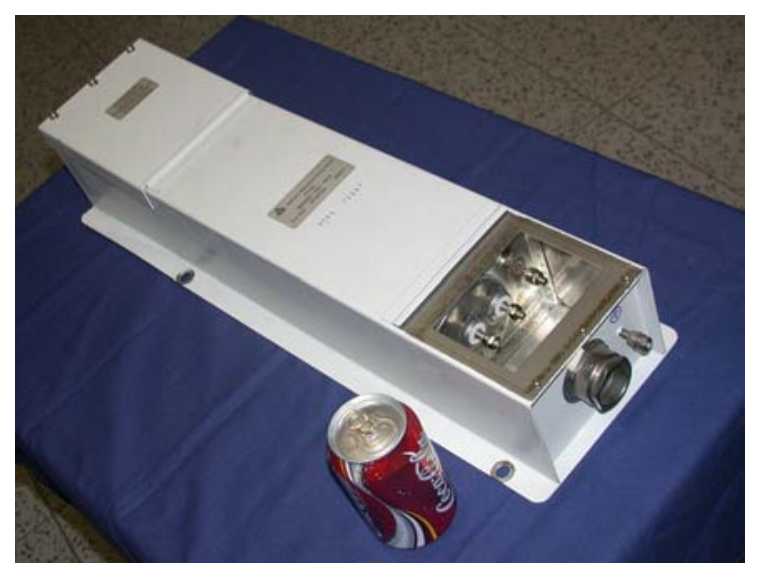

Fig. 2. Larger, 2 phase filter.

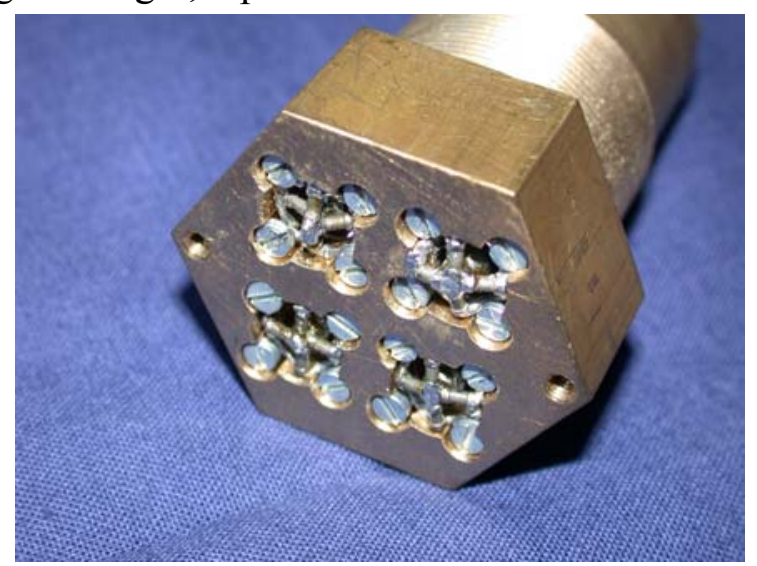

Fig. 4. 50 $\Omega-1 \Omega$ adapter - resistive elements uncovered.

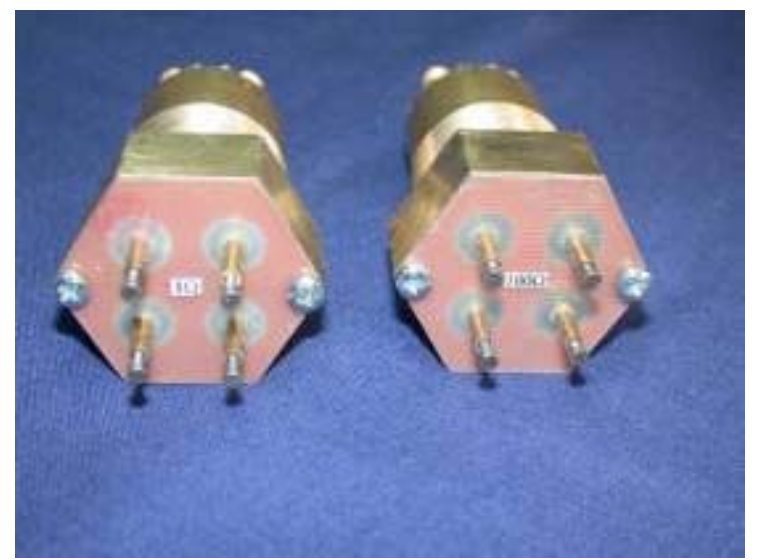

Fig. 6. Complete resistive adapters for three-phase filters 


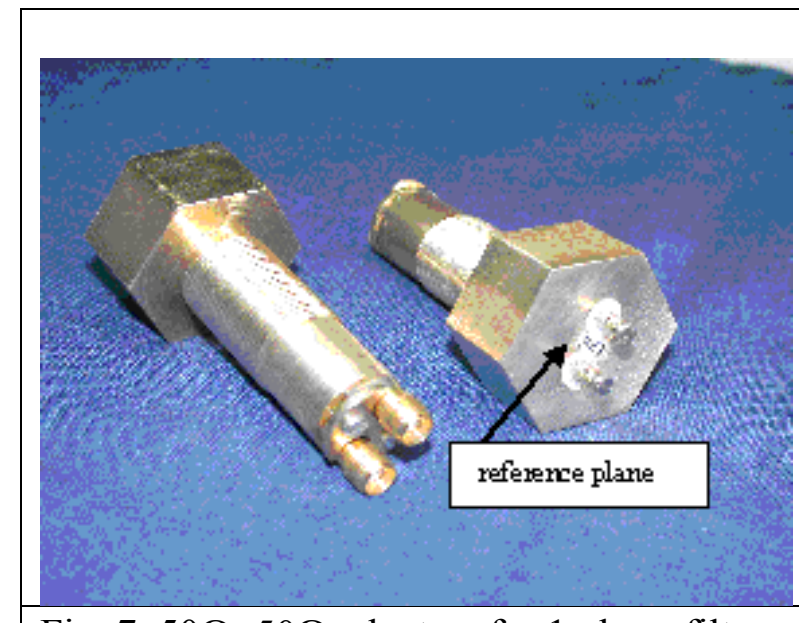

Fig. 7. $50 \Omega-50 \Omega$ adapters for 1 phase filters

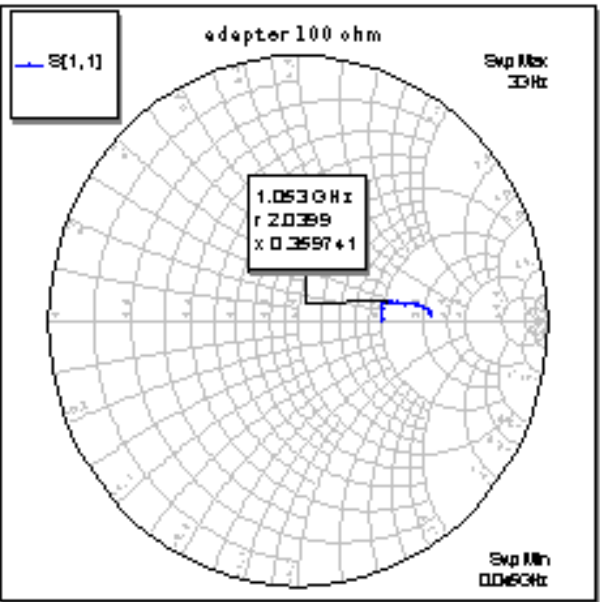

Fig. 9. Input impedance of the $50 \Omega-100 \Omega$ adapter

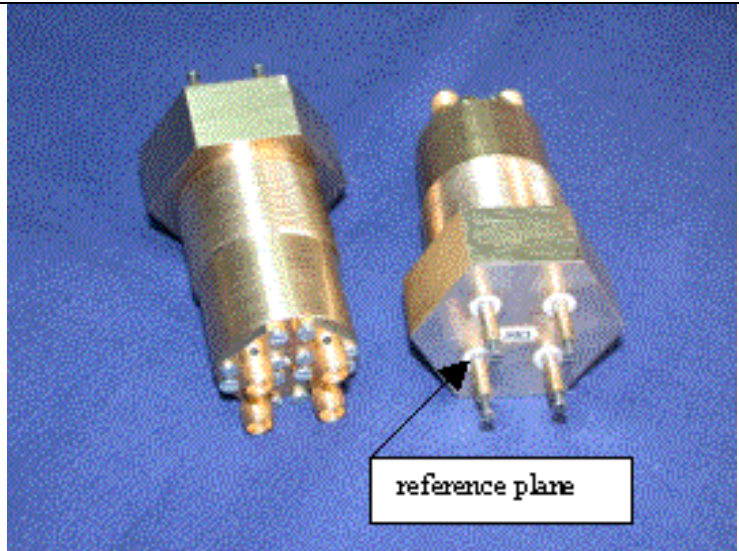

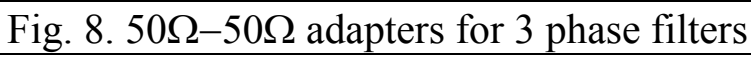

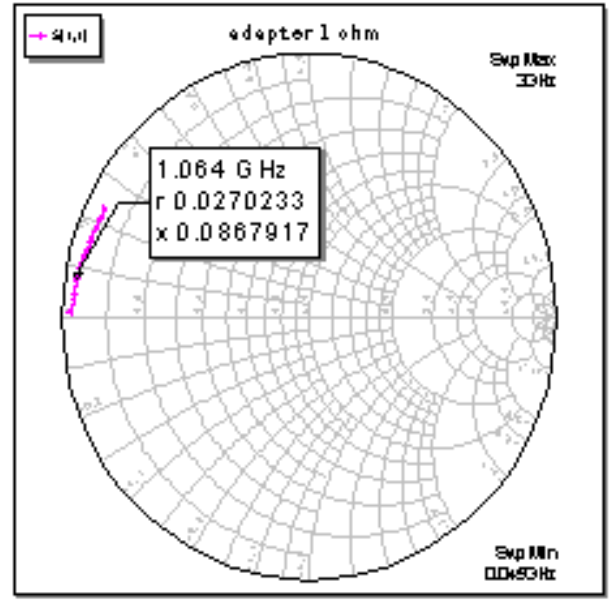

Fig. 10. Input impedance of the $50 \Omega-$ $1 \Omega$ adapter

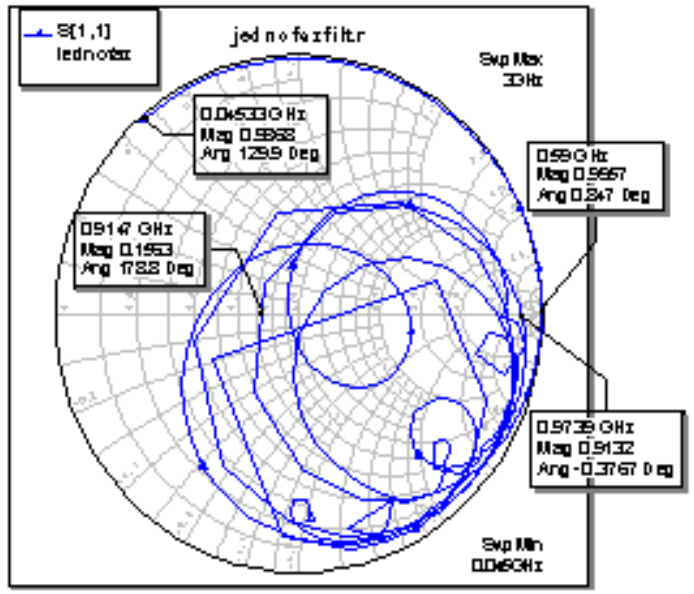

Fig. 12. Input impedance of 1 phase filter.

Fig. 11. Input impedance of data line filter. 


\section{Measurement results}

The impedances of $50 \Omega-1 \Omega$ as well as $50 \Omega-100 \Omega$ adapters were measured up to $3 \mathrm{GHz}$ from $1 \Omega$ and $100 \Omega$ side, see Fig. 9. and 10. Adapters $50 \Omega-50 \Omega$ were used to measure input impedance of filters under test. Fig. 11. to 13. show the results.

\section{Conclusion}

Some methods have been suggested [2], this is the first time real measured results are reported, along with the method used to obtain them and hardware adapters.

Measured data suggest that recommendations given in [1] are not useful in GHz band. There is no advantage in measurement in a $100 \mathrm{ohm}$ environment with respect to behavior of real filters.

\section{Acknowledgment}

This research has been supported by the Czech Grant Agency under grant no. 102/04/1079.

\section{References}

[1] European standard CISPR17: 1981

[2] Hoffmann, K and Skvor, Z.: On Radio Interference Filter Measurement. In: ARFTG conference digest, Boulder, December 4-5, 2003, pp. 227-232 